\title{
La couleur des sols : appréciation, mesure et relations avec les propriétés spectrales
}

\author{
Richard ESCADAFAL, Michel-Claude GIRARD \& Dominique COURAULT $\left({ }^{*}\right)$ \\ L.I.A., Unité de télédétection, ORSTOM, 70, route d'Aulnay, 93140 Bondy \\ (*) I.N.A.-P.G., Laboratoire de pédologie, 78850 Thiverval-Grignon
}

RÉSUMÉ

\begin{abstract}
La couleur est une donnée de sol très utilisée, aussi bien dans les travaux de terrain que dans les systèmes de classifications. Son étude se développe actuellement en relation avec les propriétés spectrales des sols et de leurs constituants, observées au laboratoire ou par télédétection.

Dans cet article, des éléments de colorimétrie sont rappelés, puis appliqués à une série de 84 échantillons de sols très diversifiés. La mise en évidence de la fréquence négligeable du métamérisme permet de modéliser les relations entre couleur et propriétés spectrales qui en sont à l'origine. Grâce à ce résultat, les couleurs de sol notées sur le terrain à l'aide du code Munsell prennent une signification physique, permettant d'aborder leur interprétation sous un jour nouveau.
\end{abstract}

Mots clés additionnels : Code Munsell, réflectance, domaine visible.

\begin{abstract}
Soil colour : evaluation, measurement and relation with spectral properties.
Colour is a soil datum which is very frequently used in field studies as well as in classification systems. Nowadays, it is analysed along with the spectral properties of soils and their constituents, which are observed in the laboratory or through remote sensing. In this paper, some elements of colorimetry were studied and applied to a series of 84 highly diversified soil samples. Metamerism occurred with negligible frequency, so it was possible to model the relations between colour and initial spectral properties. In consequence, the soil colours observed in the field through the Munsell soil charts could be given a physical meaning, which allows them to be newly interpreted.
\end{abstract}

Additional key words : Munsell soil charts, reflectance, visible spectrum.

\section{INTRODUCTION}

De nombreux noms vernaculaires de sols sont en lien avec la couleur. C'est aussi le cas des noms scientifiques qui en sont dérivés ; chernozem, par exemple, signifie terre noire.

La couleur a été retenue comme critère de classement dans de très nombreux systèmes de classification des sols; souvent dès le $2^{\mathrm{e}}$ niveau hiérarchique, à savoir la sous-classe, le grand groupe ou le sousgroupe, suivant les systèmes. C'est le cas de certaines classifications régionales des sols (SEGALEN, 1977).

Si son rôle n'est plus aussi dominant dans les classifications à vocation universelle, telles la classification française ou la Soil Taxonomy américaine, la couleur reste un élément très important de description et de discrimination des sols sur le terrain.

Ainsi, à partir d'une analyse statistique détaillée de l'information pédologique, GIRARD (1983) a montré que sur 15 variables de terrain, suffisantes pour carac- tériser un volume échantillon, 3 d'entre elles concernent la couleur.

Par contre, si l'importance de la couleur des sols n'a échappé à aucun pédologue, elle a le plus souvent été considérée comme une variable trop synthétique pour faire l'objet d'une étude en elle-même.

Plusieurs auteurs se sont intéressés aux problèmes méthodologiques liés à l'appréciation et à la mesure de la couleur des sols, et leurs conséquences sur le rôle de ce critère dans les classifications (SHIELDS et al., 1966 ; KARMANOV, 1970 ; MELVILLE \& ATKINSON, 1985).

Par ailleurs, l'étude des rapports entre les propriétés spectrales et la composition des sols se développe (GERBERMANN \& NEHER, 1979 ; KRISHNAN et al., 1980 ; STONER et al., 1980). De plus l'influence de la couleur des sols sur les mesures obtenues par télédétection suscite un intérêt croissant et fait l'objet de travaux très récents (HUETE et al., 1984 ; GIRARD, 
1985 ; Courault, 1986 ; Escadafal \& Pouget, 1986).

Dans cette perspective, ce travail est une mise au point visant à préciser la nature physique des relations entre la réflectance spectrale, les mesures colorimétriques, et la couleur des sols appréciée sur le terrain.

\section{II. ÉLÉMENTS DE COLORIMÉTRIE APPLICABLES AUX SOLS}

Chaque individu a son propre référentiel de couleurs et les termes de rouge, ocre ou brun foncé peuvent recouvrir des réalités assez différentes suivant les observateurs, sans parler des nuances que l'on peut vouloir préciser.

Pourtant l'œil humain est un instrument extraordinaire capable de couvrir une très grande gamme de niveaux de luminance, variant d'un facteur 1 à plusieurs millions (KoWALISKI, 1978) et de distinguer, suivant les spécialistes, de 100000 à 10 millions de nuances de couleurs (DELORME, 1982). Mais ces aptitudes étonnantes ne doivent pas nous faire oublier que la vision est un phénomène psycho-physique fortement dépendant du contexte et des individus. Les sensations qui en résultent ne sont pas directement comparables à des mesures absolues réalisées par un appareillage.

L'objet de la colorimétrie est précisément d'établir les relations entre perceptions visuelles et caractéristiques physiques des objets et de la lumière qui les éclaire, en spécifiant les conventions et conditions d'application. La couleur d'un objet dépend en particulier de la façon dont il renvoie la lumière qui l'éclaire. Suivant qu'il réfléchit plus ou moins certaines parties du spectre il aura une coloration ou une autre.

Nous n'envisagerons ici que le cas des couleurs de surface obtenues par réflexion de la lumière sur des objets non fluorescents (cas des sols).

Cette propriété de réflexion de la lumière en fonction de la longueur d'onde est la réflectance spectrale.

\section{A. Le système trichromatique $R, G, B$}

Le système trichromatique est basé sur le fait qu'une sensation colorée quelconque peut être reproduite par un mélange de 3 couleurs primaires: le rouge, le vert et le bleu. La Commission Internationale de l'Eclairage (C.I.E., 1931) a normalisé ce système Rouge, Vert, Bleu (Red, Green, Blue) en adoptant les longueurs d'ondes suivantes :

$$
\begin{aligned}
& \lambda(\mathrm{R})=700 \mathrm{~nm} \\
& \lambda(\mathrm{G})=546 \mathrm{~nm} \\
& \lambda(B)=436 \mathrm{~nm}
\end{aligned}
$$

Elles ont été choisies d'après les résultats d'essais empiriques montrant que c'est ce jeu qui permet de reproduire la gamme la plus étendue de couleurs.

Dans ce système une couleur quelconque peut être représentée par un vecteur $\overrightarrow{\mathrm{C}}$ de coordonnées $\mathrm{r}, \mathrm{g}, \mathrm{b}$ dans le repère $\overrightarrow{\mathrm{R}}, \overrightarrow{\mathrm{G}}, \overrightarrow{\mathrm{B}}$ :

$$
\text { (I) } \quad \vec{C}=r \cdot \vec{R}+g \cdot \vec{G}+b \cdot \vec{B} \quad \text { (cf. fig. 1) }
$$

Les unités sont choisies de telle sorte que l'on obtienne la couleur blanche lorsque $\mathrm{r}=\mathrm{g}=\mathrm{b}=1$.
L'axe médian correspond alors à l'axe des gris (du noir au blanc), c'est l'axe achromatique.

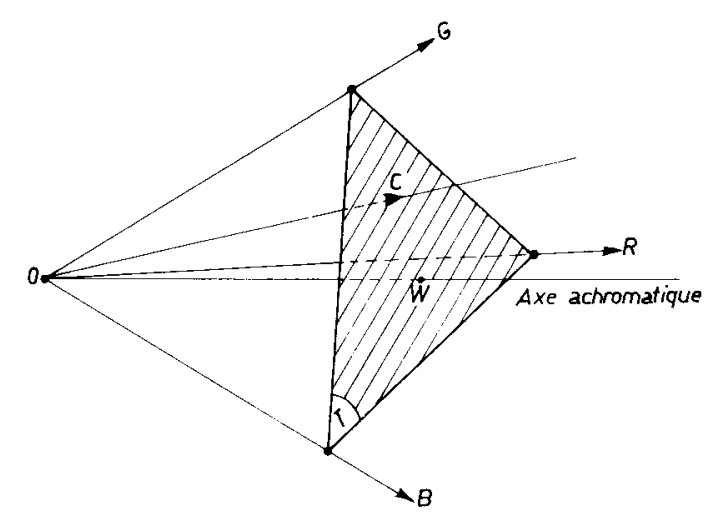

Figure 1

Représentation d'une couleur $C$ dans l'espace $R, G, B$.

Plotting of a given color $C$ in the $R, G, B$ space.

Les courbes de sensation colorées d'un observateur moyen, ou fonctions de mélange sont basées sur des séries de mesures réalisées au colorimètre avec un angle de vision compris entre $1^{\circ}$ et $4^{\circ}$. Elles définissent les caractéristiques de l'observateur de référence CIE-1931. Elles expriment les pourcentages de rouge, vert et bleu à mélanger pour obtenir la sensation correspondant à chacune des radiations monochromatiques du spectre visible. On y voit clairement apparaître un terme $r$ négatif, avec un minimum autour de $510 \mathrm{~nm}$ (fig. 2).

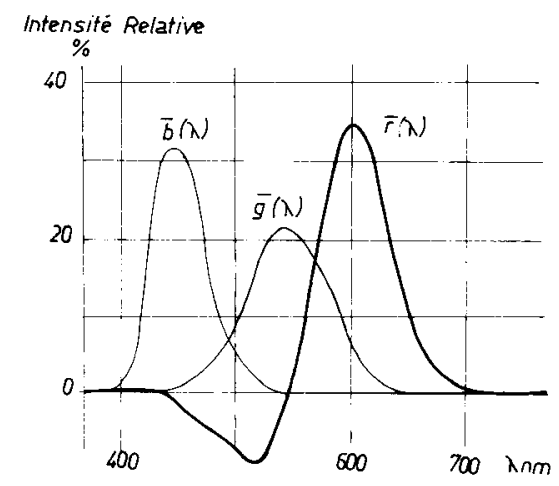

Figure 2

Courbes de reproduction des couleurs monochromatiques par mélange de rouge, vert et bleu (fonctions de mélange $r, g, b$ pour l'observateur de référence C.I.E. - 1931).

Reproducing monochromatic colours by mixing red, green and blue (colour matching functions of the C.I.E. standard observer - 1931).

L'existence des courbes de sensation colorées constitue le fondement même de la colorimétrie et a les conséquences importantes suivantes.

\section{Le calcul de la couleur d'un objet}

Puisque ces courbes permettent de calculer les composantes $R, G, B$ d'un objet théorique à pouvoir de réflexion monochromatique, en appliquant les lois 
d'additivité, on peut calculer ces mêmes composantes pour un objet réel de courbe de réflectance quelconque. Celle-ci est alors considérée comme étant la somme des valeurs des réflectances monochromatiques élémentaires sur l'ensemble des longueurs d'onde du spectre visible.

C'est ce qu'expriment les équations suivantes :

$$
\begin{aligned}
& R=k \int_{380 \mathrm{~nm}}^{780 \mathrm{~nm}} C(\lambda) \cdot H(\lambda) \cdot \overrightarrow{\mathrm{r}}(\lambda) \mathrm{d} \lambda \\
& \mathrm{G}=\mathrm{k} \int_{380 \mathrm{~nm}}^{780 \mathrm{~nm}} \mathrm{C}(\lambda) \cdot H(\lambda) \cdot \overline{\mathrm{g}}(\lambda) \mathrm{d} \lambda \\
& B=k \int_{380 \mathrm{~nm}}^{780 \mathrm{~nm}} C(\lambda) \cdot H(\lambda) \cdot \bar{b}(\lambda) \mathrm{d} \lambda
\end{aligned}
$$

$C(\lambda)$ : réflectance spectrale ;

$H(\lambda)$ : répartition spectrale d'énergie de la source lumineuse.

Ainsi, par intégration des 3 fonctions de mélange $(\overline{\mathrm{r}}(\lambda), \overline{\mathrm{g}}(\lambda), \overline{\mathrm{b}}(\lambda))$ pondérées par la courbe de réflectance d'un objet, il est possible de calculer la sensation colorée qu'il produit, sous un éclairage donné.

Pour préciser ce dernier point, et normaliser les conditions d'observation, les courbes de répartition spectrale de l'énergie de différents types de sources lumineuses ont été définies par la C.I.E. Ce sont les illuminants standards, qui comprennent notamment pour la lumière du jour le type $C$, défini en 1931 , et le type D65, recommandé plus récemment. Les tables utilisées dans les calculs colorimétriques font référence à ces standards (WYSZECKI \& STILES, 1982 ; ASTM, 1974).

En résumé, un objet de propriétés spectrales données, éclairé par une lumière de composition donnée, apparaît à un observateur moyen sous une couleur précise et une seule, que l'on peut déterminer par calcul. Ceci est bien entendu applicable aux sols.

\section{Le métamérisme}

Ce terme désigne le phénomène par lequel des objets de propriétés spectrales différentes peuvent produire la même sensation colorée.

Ainsi, un objet fortement réflectant dans le rouge et dans le vert paraîtra jaune, tout comme un objet réflectant dans les longueurs d'onde des jaunes monochromatiques.

Pour que 2 objets apparaissent de la même couleur, leurs courbes de réflectance doivent être telles que les équations (II) satisfassent les relations :

$$
\begin{aligned}
R & =k \int_{380 \mathrm{~nm}}^{780 \mathrm{~nm}} \mathrm{C} 1(\lambda) \cdot H 1(\lambda) \cdot \overline{\mathrm{r}}(\lambda) \mathrm{d} \lambda \\
& =\mathrm{k} \int_{380 \mathrm{~nm}}^{780 \mathrm{~nm}} \mathrm{C} 2(\lambda) \cdot \mathrm{H} 2(\lambda) \cdot \overline{\mathrm{r}}(\lambda) \mathrm{d} \lambda
\end{aligned}
$$

et suivant le même modèle pour $G$ et $B$.

$\mathrm{Ce}$ système d'équations est assez complexe à résoudre et les conditions du métamérisme font encore actuellement l'objet de recherches théoriques et appliquées (Ch. Golllot, comm. orale, 1987).

Sur le plan des conséquences pratiques de ce phénomène, il est important de noter que 2 couleurs ne sont généralement métamères que sous un éclairage $H(\lambda)$ donné. Dans ce cas le système d'équations (III) se simplifie, le seul terme différent entre les 2 membres étant alors la réflectance spectrale $C(\lambda)$.

L'illustration la plus intéressante pour le sujet qui nous préoccupe est le cas des échantillons de couleur des planches Munsell.

Ils sont composés à partir de mélanges de pigments reproduisant une sensation colorée. La courbe de réflectance de l'échantillon Munsell 10 YR 6/6 (brun jaunâtre) par exemple, est nettement différente de celle d'un sol qui paraît de cette couleur à la lumière du jour (fig. 3). Ces deux courbes sont métamères sous cet éclairage, mais elles ne le sont probablement pas sous un autre.

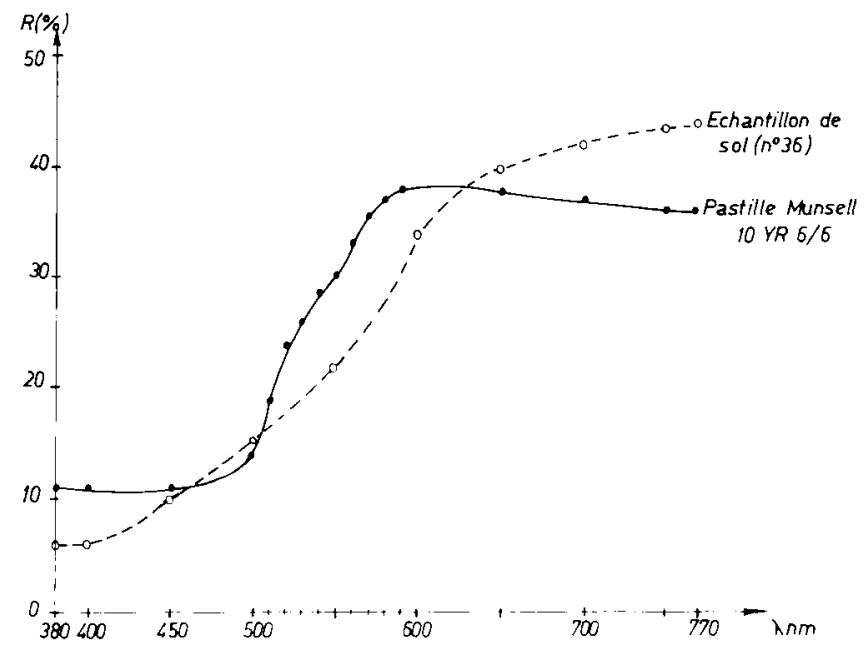

Figure 3

Courbes de réflectance d'une pastille colorée de planche Munsell et d'un échantillon de sol paraissant de la même couleur.

Example of the reflectance curves of a soil sample and of its corresponding Munsell color chip.

Ainsi, la notation de la couleur d'un échantillon de sol obtenue par comparaison avec les planches Munsell sous un éclairage artificiel, à tube fluorescents, en particulier, peut donner des résultats sensiblement différents de ceux obtenus sur le terrain.

Suivant la pureté de la couleur il existe un nombre plus ou moins grand de métamères possibles. Dans la gamme de couleurs des sols, qui sont relativement peu saturées, on peut prévoir un degré de métamérisme élevé. Des méthodes de calcul très élaborées ont été développées (TAKAHAMA \& NAYATANI, 1972), permettant notamment de prévoir le nombre de métamères pour une couleur donnée.

L'un des résultats pratiques les plus importants en ce qui nous concerne est le fait que les courbes de réflectance d'objets dont les couleurs sont métamères doivent nécessairement s'entrecroiser. L'étude théorique de cet aspect a été faite notamment par simulation (OHTA \& WYSZECKI, 1977) mettant en évidence 3 intersections, le plus souvent 4 ou 5 .

\section{B. Le système de notation des couleurs de la C.I.E.}

Le système $R, G, B$, qui a été à la base de l'étude scientifique des couleurs, présente l'inconvénient 
d'utiliser un repère quelconque et d'introduire des valeurs négatives. Par souci de rationalisation, la Commission Internationale de l'Eclairage (C.I.E.) a défini un repère cartésien basé sur les composantes trichromatiques $\mathrm{X}, \mathrm{Y}, \mathrm{Z}$, telles que :

$$
\begin{array}{lr}
X=2,7659 R+1,7519 G+1,1302 B \\
Y= & R+4,5909 G+0,06012 B \\
Z= & 0,0565 G+5,5944 B
\end{array}
$$

Ces composantes ont été choisies de telle sorte que $Y$ corresponde à la luminance d'après sa définition, de ce fait $\mathrm{X}$ et $\mathrm{Z}$ n'ont pas de réalité physique.

Les coordonnées trichromatiques $x, y, z$ sont déduites de (IV) suivant la relation :

$$
\mathrm{X} / \mathrm{X}=\mathrm{y} / \mathrm{Y}=\mathrm{Z} / \mathrm{Z}=1 / \mathrm{X}+\mathrm{Y}+\mathrm{Z} \text {. }
$$

Une couleur donnée est alors le plus souvent repérée par sa composante $\mathrm{Y}$ et ses coordonnées $\mathrm{x}$ et $\mathrm{y}$.

C'est le système scientifique international actuellement en vigueur. Du fait de ses limitations, la notation $R, G, B$ n'est généralement plus utilisée. Cependant, dans la suite de ce travail nous serons amenés dans certains cas à y retourner, car elle s'est révélée permettre une interprétation plus aisée des relations entre couleur et propriétés spectrales.

\section{III. ÉTUDE COLORIMÉTRIQUE D'UNE SÉRIE D'ÉCHANTILLONS DE SOLS VARIÉS}

\section{A. Calcul de la couleur d'après les courbes de réflec- tance spectrale}

Les calculs colorimétriques reposent sur la détermination de la courbe de réflectance spectrale des objets étudiés. Ceci peut être réalisé au laboratoire, sur des échantillons de $1 \mathrm{~cm}^{2}$ environ, en utilisant un spectrophotomètre.

La méthode standardisée par la C.I.E. utilise une sphère intégratrice pour éliminer tout rayonnement parasite et réaliser une mesure rigoureuse. Cette méthode est utilisée dans l'industrie et les laboratoires pour toutes les déterminations colorimétriques, elle a déjà été appliquée aux sols (SHIELDS et al., 1966) et aux roches (CERVELLE et al., 1977).

Dans ce travail nous avons étudié les courbes de réflectance d'un ensemble de 84 échantillons de sols de couleur et de composition très variées collectés à l'occasion de travaux pédologiques réalisés en France et dans le monde. Elles ont été mesurées avec un spectrophotomètre Beckmann DK2 sur échantillons séchés à l'air et tamisés à $2 \mathrm{~mm}$, placés dans des boîtes à couvercle de verre.

Toutes les courbes de réflectance de ces échantillons ont en commun la caractéristique d'être toujours croissantes dans le domaine visible. La pente en est généralement faible au début, elle peut croître ensuite régulièrement ou assez brutalement, puis s'infléchir ou non. Dans tous les cas, la pente n'est jamais négative (fonction monotone croissante, au sens large).

Ces observations rejoignent celles de COMBE (1984) et Traube (1985). CONDIT (1970), qui a réalisé l'analyse statistique des courbes de réflectance de 285 échantillons représentant un large éventail de sols des U.S.A., a montré de même que les courbes observées sont toutes croissantes dans la gamme visible.

En appliquant des équations similaires à (II), on peut calculer la couleur correspondant à chacune de ces courbes et obtenir les coordonnées $\mathrm{Y}, \mathrm{x}, \mathrm{y}$ de chaque échantillon de sol sous un éclairage donné. C'est ce que nous avons fait avec un programme de calcul utilisant les valeurs de $H(\lambda) \cdot \bar{x}(\lambda), \quad H(\lambda) \cdot \bar{y}(\lambda)$ et $H(\lambda) \cdot \bar{z}(\lambda)$ pour l'illuminant $C$, suivant un pas de $10 \mathrm{~nm}$ (CERVELLE et al., 1977).

\section{B. Modèle d'inversion de la relation réflectance- couleur}

Si donc le calcul de la relation entre propriétés spectrales des sols dans le domaine visible et aspect coloré ne pose pas de difficultés, par contre nous avons vu que le phénomène du métamérisme s'oppose en revanche à l'inversion de cette relation. Il n'est théoriquement pas possible de prévoir le comportement spectral d'un objet d'après sa couleur.

Le cas des sols est cependant un peu particulier du fait que les courbes de réflectance sont monotones croissantes dans le visible. Il en découle que la probabilité que 2 sols de même couleur présentent des courbes de réflectance entrecroisées est très faible. En d'autres termes, on peut estimer que le phénomène du métamérisme a peu de chances de se produire dans le cas des sols. Ceci laisse espérer de pouvoir établir une relation bijective entre couleur et propriétés spectrales.

Pour vérifier cette hypothèse, nous avons testé les corrélations multiples entre la réflectance spectrale $\operatorname{RF}(\lambda)$ aux différentes longueurs d'onde $(\lambda)$, et les coefficients trichromatiques calculés. Le modèle faisant intervenir les coefficients calculés $\mathrm{Rc}, \mathrm{Gc}, \mathrm{Bc}$ (déduits de $\mathrm{X}, \mathrm{Y}, \mathrm{Z}$ en inversant la relation (IV)) s'est révélé le plus intéressant :

$$
\mathrm{RF}(\lambda)=\mathrm{a} \lambda \cdot \mathrm{Rc}+\mathrm{b} \lambda \cdot \mathrm{Gc}+\mathrm{c} \lambda \cdot \mathrm{Bc}+\mathrm{d} \lambda
$$

$\mathrm{a} \lambda, \mathrm{b} \lambda, \mathrm{c} \lambda, \mathrm{d} \lambda=$

coefficients des régressions linéaires multiples.

Pour les longueurs d'onde de 400 à $750 \mathrm{~nm}$ échantillonnées tous les $50 \mathrm{~nm}$, nous avons obtenu, sur notre série de 84 échantillons, les coefficients qui sont reportés dans le tableau 1.

On remarque premièrement la forte liaison entre les coefficients $R, G, B$ et la réflectance dans les lon-

\section{TABLEAU}

Coefficients des régressions linéaires multiples entre réflectance $R F(\lambda)$ et couleur $R c, G c, B c$.

Multiple linear regression coefficients between reflectance $R F(\lambda$ and colour $R c, G c, B c$

\begin{tabular}{cccccc}
\hline \hline$\lambda(\mathrm{nm})$ & $\mathrm{a} \lambda$ & $\mathrm{b} \lambda$ & $\mathrm{c} \lambda$ & $\mathrm{d} \lambda$ & $\mathrm{r}$ (mult.) \\
\hline 400 & 0 & 0 & 0,0393 & $-0,521$ & 0,985 \\
450 & 0 & 0 & 0,0477 & $-0,120$ & 0,999 \\
500 & 0 & 0,0190 & 0,0356 & $-0,075$ & 0,999 \\
550 & 0,0046 & 0,0477 & 0,0046 & $-0,020$ & 0,998 \\
600 & 0,0498 & 0,0062 & 0,0059 & $-0,239$ & 0,999 \\
650 & 0,0719 & $-0,0248$ & 0,0181 & $+0,435$ & 0,998 \\
700 & 0,0801 & $-0,0345$ & 0,0225 & $+1,558$ & 0,995 \\
750 & 0,0868 & $-0,0544$ & 0,0371 & $+4,242$ & 0,980 \\
\hline
\end{tabular}

(avec $\mathrm{Rc}, \mathrm{Gc}, \mathrm{Bc} \times 10^{4}$ ). 
gueurs d'onde correspondant respectivement au rouge, au vert et au bleu (importance des termes a $\lambda$ à $650 \mathrm{~nm}, \mathrm{~b} \lambda$ à $550 \mathrm{~nm}$ et $\mathrm{c} \lambda$ à $450 \mathrm{~nm}$ ). C'est cette intéressante liaison qui nous a incités à exprimer les couleurs dans le système $R, G, B$.

De plus, les coefficients de corrélation très élevés obtenus montrent qu'il est possible de reconstruire la courbe de réflectance spectrale à partir des valeurs $R$, G, B. L'écart moyen entre la valeur observée et modélisée est de 0,5 p. 100 pour les longueurs d'onde de 500 à $600 \mathrm{~nm}$, et de 1,5 p. 100 aux extrémités du spectre, autour de 400 et de $700 \mathrm{~nm}$.

La figure 4 illustre ce résultat avec 5 courbes représentatives de la variété des échantillons étudiés. Elles montrent que pour l'ensemble des échantillons la courbe modélisée suit presque parfaitement la courbe réelle.

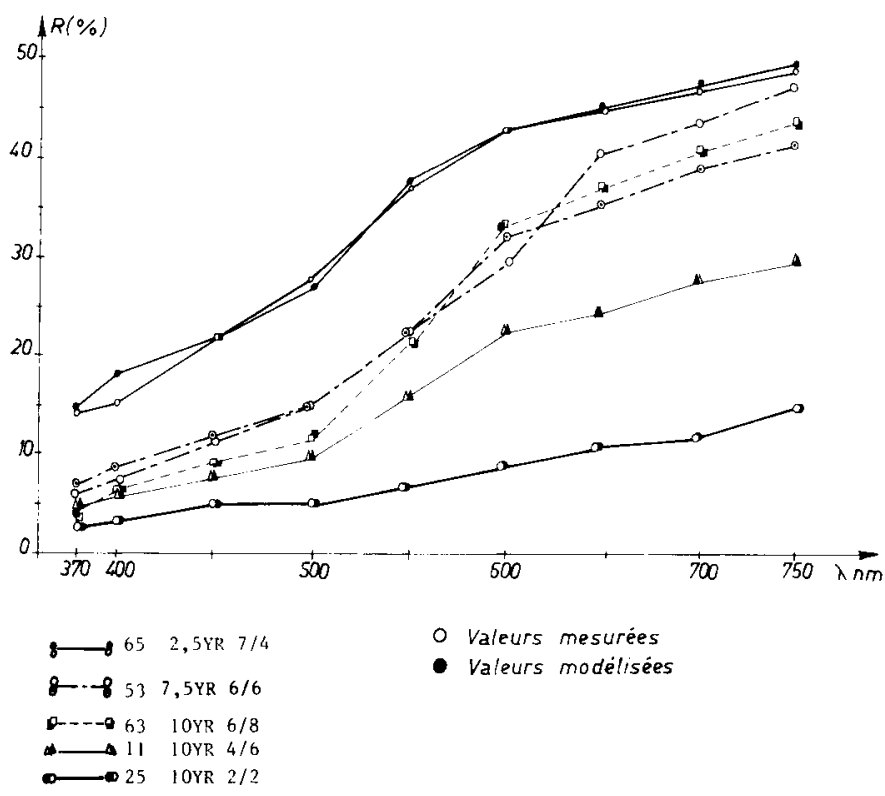

Figure 4

Courbes de réflectance de 5 échantillons de sols représentatifs de la gamme étudiée (valeurs mesurées et modélisées).

Spectral reflectance curves for 5 soil samples extracted from the studied set (observed and predicted values).

Sur les 84 cas, un seul ( $n^{\circ} 53$ ) présente un écart important entre valeur observée et valeur modélisée pour les longueurs d'onde 600 à $750 \mathrm{~nm}$. Or, les valeurs $R, G, B$ correspondant à la courbe modélisée et celles obtenues pour la courbe mesurée sont identiques. Autrement dit, bien que la courbe de réflectance de l'échantillon $n^{\circ} 53$ soit nettement différente de la courbe attendue, elle produit la même sensation colorée ; il s'agit là du seul cas de métamérisme que nous ayons rencontré.

Nous avons donc montré jusqu'ici, comment appliquer les calculs colorimétriques aux sols et dans quelles conditions, en relation avec la très faible fréquence du métamérisme, les relations entre les propriétés spectrales des sols et leurs couleurs peuvent être inversées dans la majorité des cas.

\section{L'APPRÉCIATION \\ DE LA COULEUR DES SOLS SUR LE TERRAIN}

La détermination précise de la couleur des sols requiert des mesures spectrophotométriques en laboratoire, qui actuellement, ne sont pas réalisées en routine. En revanche, les pédologues apprécient et notent systématiquement la couleur lorsqu'ils décrivent les fosses et sondages sur le terrain.

\section{A. L'utilisation du système Munsell}

La nécessité de codifier cette appréciation visuelle de la couleur en se basant sur des comparaisons avec des étalons colorés s:est rapidement faite sentir dès les débuts de l'étude des sols, car la couleur apparaissait déjà comme une donnée importante.

Après diverses tentatives dont l'utilisation de disques rotatifs en 1924, les pédologues américains en sont arrivés à l'idée de réaliser une gamme de couleurs étalons adaptées aux couleurs de sols les plus fréquemment rencontrées (PENDLETON \& NICKERSON, 1951). Ceci a été réalisé en collaboration avec la société Munsell, qui, après avoir édité un des premiers atlas de couleur destinés à l'industrie des colorants, a commercialisé un ensemble de planches de couleur de sols (Munsell Soil Color Charts, 1950).

Suivant les recommandations de l'U.S. Department of Agriculture, l'utilisation de ces planches Munsell a été adoptée par l'ensemble des pédologues américains, puis à leur suite par une grande partie de leur collègues. Leur usage est toujours en vigueur dans la communauté pédologique internationale, y compris en France (JAMAGNE, 1967).

Le système Munsell est organisé d'une façon qui paraît assez naturelle, les couleurs y sont hiérarchisées en distinguant d'abord la teinte (hue, en anglais), puis la clarté (value), et enfin la pureté (chroma), qui exprime la saturation de la couleur.

A chaque planche correspond une teinte, elle présente des échantillons de clarté croissante en ordonnée et de pureté (ou saturation) croissante en abscisse (fig. 5). Pour les planches de couleur de sols, la gamme des clartés s'étend de 2,5 à 8 , et la pureté de 1 à 8 , contre respectivement 0 à 10 et 0 à 20-24 dans la gamme maximum théorique.

La couleur du sol est comparée le plus souvent sur le terrain, à la lumière du jour, avec les couleurs des planches. En déplaçant l'échantillon de sol sous les fenêtres prévues sous chaque échantillon coloré, on repère celui dont la couleur se rapproche le mieux et on note ses coordonnées (teinte, clarté, pureté).

La précision de cette méthode de détermination des couleurs dépend beaucoup du soin que l'on y apporte, comme l'ont rappelé récemment MELVILLE \& ATKINSON (1985).

Pour minimiser les erreurs d'appréciation de la couleur par comparaison avec les planches, il faut s'entourer d'un certain nombre de précautions qu'il peut être utile de résumer ici.

Dès lors que l'on entreprend d'estimer visuellement des couleurs, il semble évident qu'il faut d'abord s'assurer qu'on en a les capacités physiologiques. En 


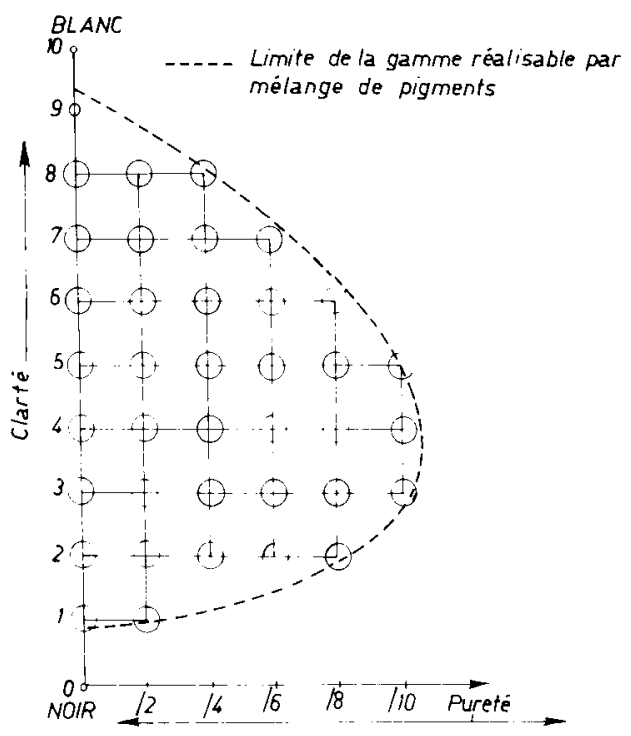

Figure 5

Organisation des couleurs sur une planche Munsell (teinte constante).

Distribution of the colors on a Munsell chart (constant hue).

effet, les anomalies plus ou moins fortes de la vision des couleurs constatées chez les hommes (avec une fréquence d'environ 6 p. 100 , contre 0,2 p. 100 chez les femmes) concernent particulièrement la gamme des teintes orangées, qui est typiquement celle des sols. Des tests de dépistage existent et leur utilisation est vivement recommandée par les spécialistes en colorimétrie.

Ensuite, les conditions d'observation doivent être contrôlées au mieux. Sur le terrain, on choisira par exemple d'opérer sous un ciel clair, en s'orientant vers le nord et en évitant les heures de début et de fin de journée. Si l'on recherche la meilleure précision et la meilleure reproductibilité possible, il faudra recourir à l'observation au laboratoire, sous un éclairage artificiel normalisé.

Dans tous les cas, il faut enfin s'assurer que l'environnement visuel est neutre, notamment que les murs, vêtements ou objets à proximité ne sont pas trop colorés pour ne pas perturber la composition spectrale de la lumière incidente sur la planche de couleurs.

L'ensemble de toutes les teintes du système Munsell se répartit sur un cercle, mais l'atlas des couleurs de sols ne contient que les teintes rouges à jaune verdâtre (10 R à $5 \mathrm{GY}$ ). L'axe achromatique figurant la gamme des gris passe par le centre. Pour une clarté donnée, on observe à la périphérie les teintes saturées et vers le centre les couleurs de pureté décroissante. Dans ce système une couleur donnée est caractérisée par des coordonnées de type cylindrique (fig. 6).

\section{B. Les relations entre le système Munsell et le système C.I.E.}

Nous avons vu que dans le système $X, Y, Z$ de la C.I.E. les couleurs sont repérées suivant des coordonnées cartésiennes. Un changement de repère pourrait donc permettre le passage d'un système de notation des couleurs à l'autre.

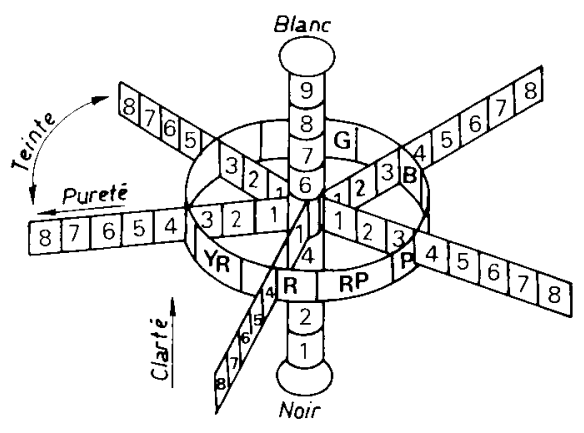

figure 6

L. 'espace des couleurs Muthell et ses coordonnees cylindriques. The cylindrical coordinates of the Munsell color system.

En fait, ce n'est pas si simple, car le système Munsell a des limitations importantes liées à ses origines. Notons premièrement que la nature des pigments disponibles, ne permet pas de faire figurer la gamme complète des couleurs dans l'atlas. Dans le cas des couleurs de sols cette limitation ne semble pas exister, mais certaines couleurs peu fréquentes ne sont pas représentées.

Il faut surtout rappeler que le système a été mis au point de façon empirique par Munsell de telle sorte que l'écart de couleur perçu entre les différents échantillons de couleurs paraisse régulier, satisfaisant ainsi les besoins des coloristes. Les travaux de la C.I.E. et en particulier ceux de Mac ADAM ont montré, au contraire, que dans les espaces $R, G, B$ et $X, Y, Z$ les distances perçues entre couleurs ne suivent pas une métrique euclidienne, mais riemannienne (KowALISKI, 1978). En d'autres termes, la géométrie de l'espace des couleurs Munsell et celle du système international ne sont pas de même nature, il n'est pas possible de passer de l'un à l'autre par un simple jeu d'équations.

Le passage entre la notation Munsell et le système C.I.E. est basé sur la mesure de la courbe de réflectance de chaque pastille des planches de couleurs et le calcul des coefficients $\mathrm{x}, \mathrm{y}, \mathrm{Y}$ pour l'éclairage sous lequel les planches sont observées.

Ces calculs ont été réalisés pour un éclairage de type illuminant $\mathrm{C}$ et sont disponibles sous forme de tables de conversion et d'abaques qui permettent de passer d'un système à l'autre (WISZECKI \& STILES, 1982 ; ASTM, 1974).

\section{Comparaison entre les couleurs calculées et les couleurs appréciées sur le terrain}

Nous avons cherché à exploiter cette possibilité pour comparer les coefficients $\mathrm{Rc}, \mathrm{Gc}, \mathrm{Bc}$ calculés précédemment pour chacun de nos 84 échantillons, avec les couleurs appréciées sur le terrain en utilisant le code Munsell. Après conversion de ces dernières en notation C.I.E., on obtient les coefficients « appréciés »: $\mathrm{Ra}, \mathrm{Ga}, \mathrm{Ba}$.

Les corrélations linéaires obtenues entre la série des valeurs calculées et la série des valeurs observées sont les suivantes :

$$
\begin{array}{ll}
\mathrm{Rc}=0,713 \mathrm{Ra}+0,00821 & \mathrm{r}=0,95 \\
\mathrm{Gc}=0,766 \mathrm{Ga}+0,00693 & \mathrm{r}=0,94 \\
\mathrm{Bc}=0,764 \mathrm{Ba}+0,0078 & \mathrm{r}=0,91
\end{array}
$$


La couleur Munsell ayant été notée pär valeurs entières de clarté et de pureté, on compare donc des valeurs discrètes avec des valeurs continues. En conséquence, on peut considérer que les résultats des corrélations sont satisfaisants.

Notons également qu'une erreur d'une unité sur la luminance entraîne une erreur sur les coefficients $\mathrm{Ra}$, $\mathrm{Ga}, \mathrm{Ba}$ d'autant plus forte que les sols sont plus clairs, car la luminance varie avec le carré de la clarté.

Enfin, la détermination des couleurs en utilisant les planches Munsell s'est faite à la lumière du jour réelle, dont on sait que la composition est assez fluctuante, et non avec l'illuminant $C$. Ceci peut expliquer la dérive systématique constatée entre les valeurs calculées $(\mathrm{Rc}, \mathrm{Gc}, \mathrm{Bc})$ et appréciées ( $\mathrm{Ra}, \mathrm{Ga}, \mathrm{Ba})$.

Cette comparaison a montré que les couleurs notées sur le terrain avec le code Munsell permettent d'obtenir une bonne approximation des coefficients trichromatiques déterminés normalement au laboratoire.

Or nous avons démontré que, à partir de ces mêmes coefficients trichromatiques, nous pouvons obtenir, par modélisation, une excellente estimation de la courbe de réflectance spectrale des sols.

Ces 2 derniers points nous conduisent à conclure que le simple fait de noter la couleur du sol sur le terrain peut nous permettre d'en approcher les propriétés spectrales dans le domaine visible.

\section{CONCLUSION}

Pour préciser les bases physiques de l'étude des relations entre la couleur et les autres données de sol, nous avons appliqué un certain nombre de principes et calculs colorimétriques à une série de 84 échantillons de sol très diversifiés.
Ce travail a montré que la donnée "couleur du sol » saisie sur le terrain peut être interprétée beaucoup plus finement que la simple utilisation des données brutes exprimées en teinte, clarté et pureté.

En effet, comme le métamérisme se produit peu souvent, nous avons pu modéliser, à partir de la couleur, les propriétés spectrales du sol qui en sont à l'origine.

L'estimation de la couleur du sol est une donnée facile à obtenir et dont la fiabilité peut être bonne si l'on s'entoure d'un certain nombre de précautions. Elle est en particulier largement disponible dans les notices de très nombreuses cartes pédologiques.

Sans atteindre la précision des mesures spectrophotométriques faites au laboratoire, la couleur permet donc de caractériser rapidement le comportement spectral d'un sol dans le domaine visible. Ces résultats sont actuellement utilisés en télédétection et appliqués à l'étude des rapports entre la couleur, la nature et l'organisation des constituants des sols.

D'une façon plus générale, nous espérons que ce travail, en donnant un sens physique à la notation Munsell traditionnellement utilisée par les pédologues, contribuera à simplifier l'interprétation de la nature et du rôle de la couleur des sols.

Recu le 9 juillet 1987. Accepté le 12 novembre 1987.

\section{REMERCIEMENTS}

Nous remercions vivement M. Cervel.I.E, Directeur de Recherches au laboratoire de Minéralogie-cristallographie de l'Université Pierre et Marie-Curie (Paris VI), qui nous a communiqué le programme de calcul des coefficients trichromatiques, et M. GollLOT, professeur de physique à l'Institut National Agronomique Paris-Grignon, pour ses conseils en matière de colorimétrie.

\section{RÉFÉRENCES BIBLIOGRAPHIQUES}

A.S.T.M., 1974. Annual book of A.S.T.M. 1974. Philadelphia U.S.A. D 1535-68 reapproved 1974, $22 \mathrm{p}$.

Cervelle B., Malezieux J. M., Caye R., 1977. Expression quantitative de la couleur liée à la réflectance diffuse de quelques roches et minéraux. Bull. Soc. Fr. Mineral. Cristallogr., 100, 185-191.

C.I.E., 1932. C.I.E. proceedings 1931. Cambridge University Press, Cambridge.

Combe P., 1984. Caractérisations radiométriques de volumes pédologiques de référence. D.E.A. Paris VII-INA-PG-ORSTOM, 83 p.

Condit H. R., 1970. The spectral reflectance of American soils. Photogramm. Eng., 36, 955-966.

Courault D., 1986. Modélisation du comportement spectral de la surface du sol. Etude intraparcellaire. D.E.A. de Science des sols, INA-PG, Paris, $102 \mathrm{p}$.

Delorme A., 1982. Psychologie de la perception. Interéditions, Paris, $421 \mathrm{p}$.

Escadafal R., Pouget M., 1986. Luminance spectrale et caractères de la surface des sols en région aride méditerannéenne (Sud Tunisien). I.T.C. Journal, 19-23.

Girard M. C., 1983. Recherche d'une modélisation en vue d'une représentation spatiale de la couverture pédologique. Application à une région des plateaux jurassiques de Bourgogne. Thèse de doctorat d'Etat de l'Université Paris VII. Sols, n 12 , Paris, $490 \mathrm{p}$.
Girard M. C., 1985. Télédétection de la surface du sol. In : "Applications de la télédétection à l'agriculture », Colloques I.N.R.A., n $32,177-193$.

Gerbermann A. H., Neher D. D., 1979. Reflectance of varying mixtures of clay and sand. Photogramm. Eng. Rem. Sens., 45, 1145 1151 .

Huete A. R., Post D. F., Jackson, R. D., 1984. Soil spectral effect on 4-space vegetation discrimination. Rem. Sens. of. Env., 15, 155165 .

Jamagne M., 1967. Bases et techniques d'une cartographie des sols. I.N.R.A., Paris, $142 \mathrm{p}$.

Karmanov I. I., 1970. Study of soil from the spectral composition of reflected radiation. Soviet Soil Science, 4, 226-238.

Kowalisky P., 1978. Vision et mesure de la couleur. Masson, Paris, $197 \mathrm{p}$.

Krishnan P., Alexander J. D., Butler B. J., Hummel J. W., 1980. Reflectance technique for predicting soil organic matter. Soil Sci. Soc. Am. J., 44, 1282-1285.

Melville M. D., Atkinson G., 1985. Soil colour : its measurement and its designation in models of uniform color space. I. Soil Science, 36, 495-512.

Munsell Color Company, 1950. Munsell soil color charts. Baltimore, $7 \mathrm{pl}$. 
Ohta N., Wyszecki G., 1977. Location of the nodes of metameric color stimuli. Color Res. Appl., 2, 183-186.

Pendleton R. L., Nickerson D., 1951. Soil colors and special soil color charts. Soil Science, 71, 35-43.

Segalen P., 1977. Les classifications des sols. Revue critique. Orstom, Bondy, $175 \mathrm{p}$.

Shields J. A., St Arnaud R. J., Paul E. A., Clayton J. S., 1966. Measurement of soil color. Can. J. Soil Sc., 46, 83-90.

Stoner E. R., Baumgarner M. F., Biehl L. L., Robinson B. F., 1980. Atlas of soil reflectance properties. L.A.R.S., Purdue University, $75 \mathrm{p}$.
Takahama K., Nayatani Y., 1972. New methods for generating metameric stimuli of object color. J. Opt. Soc. Am., 62, 1516-1520.

Traube L.., 1985. Analyses radiométriques et caractérisation des différences locales de l'état de la surface des formations loessiques de la région d'Ermenonville. D.E.A. Paris VII-INA-PG-ORSTOM, $121 \mathrm{p}$.

Wyszecki G., Stiles W. S., 1982. Color science: concept and methods, quantitative data and formulae. Wiley, New York, 2nd edition, $950 \mathrm{p}$. 